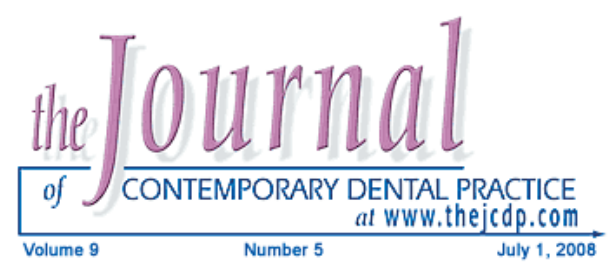

\title{
Clinical Evaluation of Dose Reduction on Image Quality of Panoramic Radiographs
}

\author{
Meryem Toraman Alkurt, DDS, PhD; Ilkay Peker, DDS, PhD; \\ Gülten Usalan, DDS, PhD; Bülent Altunkaynak, BDS, PhD
}

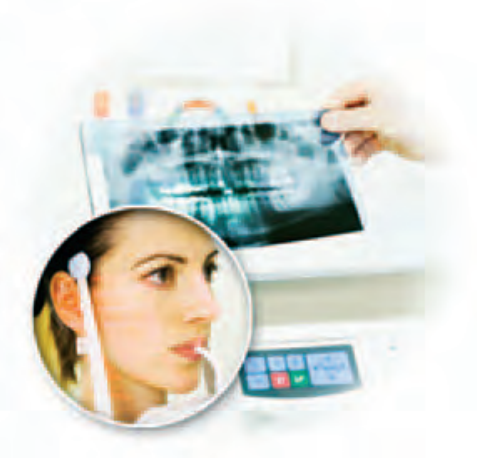

\begin{abstract}
Aim: The purpose of this study was to evaluate the effect of tube current reduction on image quality using medium and regular intensifying screens as well as a digital system for panoramic radiography.

Methods and Materials: A total of 150 panoramic images of 75 patients were obtained in the study. The initial images were taken at standard exposure settings, and secondary images were exposed with the tube current reduced at different rates.

Results: There was no statistically significant difference $(p>0.05)$ between the two exposures for Group 3 (the rate of dose reduction $25 \%$ ) while a statistically significant difference $(p<0.05)$ was found in Group 4 (the rate of dose reduction $50 \%$ ) using medium intensifying screens for all observers. No statistically significant difference was found between the two exposures on digital panoramic images.

Conclusion: According to the results of this study a dose reduction of $25 \%$ was achieved for medium intensifying screens and for digital panoramic images without any loss of image quality.

Clinical Significance: A substantial reduction in radiation exposure can be achieved in conventional panoramic radiography using a medium intensifying screen and in digital panoramic radiography without any loss of image quality needed for radiological evaluation of anatomical structures and pathological conditions.

Keywords: Panoramic radiography, dose reduction, intensifying screens, digital radiography

Citation: Alkurt MT, Peker I, Usalan G, Altunkaynak B. Clinical Evaluation of Dose Reduction on Image Quality of Panoramic Radiographs. J Contemp Dent Pract 2008 July; (9)5:034-041.
\end{abstract}

(c) Seer Publishing 


\section{Introduction}

Dose reduction within the limits of optimum image quality is a goal of radiography in order to minimize radiation exposure to patients, radiology staff, and the environment. Dose reduction can be achieved through the manipulation of tube potential (measured in $\mathrm{kV}$ ) and tube current (measured in $\mathrm{mA}$ ) and by using different screenfilm systems as well as the use of digital imaging. ${ }^{1}$

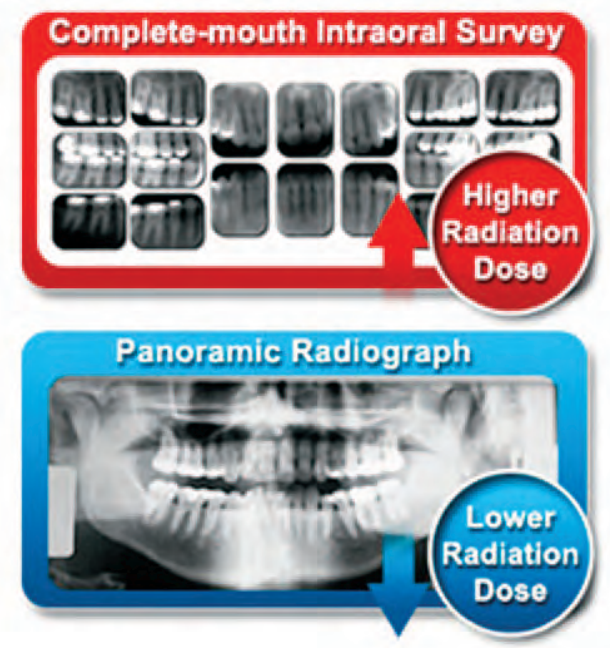

Panoramic radiographs are widely used to obtain a comprehensive survey of the maxillofacial complex. One of the advantages is reduction in radiation dose compared with a complete-mouth intraoral survey. ${ }^{2}$ Film-screen systems using a medium intensifying screen (speed class 200) have been accepted in extraoral radiography while the regular intensifying screen (speed class 400) has gained uniform acceptance in the field of maxillofacial radiology. Alteration of tube potential and tube current can reduce the radiation dose but it can also result in poorer image quality. ${ }^{1}$

Digital imaging was first introduced in dentistry for intraoral radiography but is now widely available for panoramic radiography based on either a charged couple device (CCD) or storage phosphor receptor. ${ }^{3}$ The advantages of digital techniques compared with film techniques are rapid transmission of images, the small storage space for images needed, and a lower contamination of the environment. ${ }^{4}$ Previous studies have demonstrated it is possible to achieve a degree of dose reduction in digital panoramic radiography. ${ }^{5,6,7}$

The purpose of this study was to evaluate the effect of tube current reduction on image quality

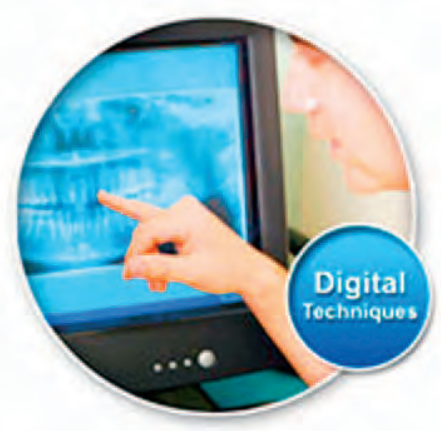

using medium and regular intensifying screens as well as a digital system for panoramic radiography.

\section{Methods and Materials}

This study had been approved by the Ethics Committee of Gazi University, Faculty of Dentistry in Ankara, Turkey in order to satisfy the Helsinki Declaration.

Seventy-five patients ( 41 female and 34 male) who required panoramic radiography for diagnostic purposes participated in the study. Exclusion criteria were pregnancy, age 17 years or less, occupational X-ray exposure, and patients with previous extensive radiographic examinations. The patients were divided into five groups with each having 15 subjects. Two panoramic images were taken of each of the 75 patients (150 radiographs) on separate days. The first image was taken at standard exposure settings and the second image was taken with the tube current reduced at different rates.

Conventional panoramic radiographs were obtained with a Trophy OP100 (Instrumentarium, Tuusula, Finland) panoramic unit. Medium (Eastman Kodak Co, Rochester, NY, USA) and regular (Dr. Goos Suprema GmbH, Heidelberg, Germany) intensifying screens $(15 \times 30 \mathrm{~cm}$ cassette) and Kodak T Mat G films (Eastman Kodak Co, Rochester, NY, USA) were used in the study. Film radiographs were developed in an automatic film processor (Velopex, Extra-X, Medivance Instruments Ltd, London, UK and NW107A) using freshly prepared processing chemicals.

Digital panoramic images were obtained with an Orthoralix 9200 DDE (Gendex Co, Milan, Italy) panoramic unit which is a CCD-based system used with VixWin 2000 software (Gendex Co, Milan, Italy). The images were assessed on the 
monitor using an 8-bit resolution. The screens, digital system, and $\mathrm{mA}$ settings used for each patient group are listed in Table 1.

All radiographs were assessed by three oral radiologists with at least ten years of experience each. The observers evaluated the images using a three-point scale ${ }^{1}(1=$ well visible, $0=$ partly visible, and $-1=$ not or hardly visible) for anatomical structures and pathological findings (Table 2) which are commonly found on panoramic radiographs.

The film radiographs were assessed using a $\times 2$ magnification X-viewer (Luminosa, CSN Industrie, Italy) in a quiet room with subdued ambient lighting. Images from the digital system were displayed directly on a 17 inch monitor screen in the same ambient lighting. To avoid observer fatigue, an interval of at least one week separated each viewing session.

\section{Data Analysis}

SPSS-version 15.0 for Windows (SPSS Inc., Chicago, IL, USA) was used for all calculations. Image quality of the groups and comparison of the observers were assessed by using the nonparametric Kruskal-Wallis and Mann-Whitney $U$ tests. The Mann-Whitney $U$ test was used to compare the two exposures with the anatomic structures and pathological findings for each group and observer. The Kruskal-Wallis test was used to compare observers in the two exposures and the anatomic structures and pathological findings. The level of statistical significance was $a=0.05$.
Results

Seventy-five patients with a mean age of 21.60 participated in the investigation from which a total of 150 radiographs were obtained for the study.

There was no statistically significant difference ( $p>0.05$ ) between the two exposures in terms of image quality of anatomical structures, but a statistically significant difference $(p<0.05)$ in image quality was found between the two exposures of pathological findings for Group 1 and the first observer. For Group 1 and second observer, there was a statistically significant difference $(p<0.05)$ in image quality between the two exposures of both anatomical structures and pathological findings. For Group 1 and third observer, there was no statistically significant difference $(p>0.05)$ in image quality between the two exposures of either anatomical structures or pathological findings.

For Group 2, a statistically significant difference $(p<0.05)$ in image quality was found between the two exposures of both anatomical structures and pathological findings for the first and second observers. For Group 2 and the third observer, there was no statistically significant difference $(p>0.05)$ in image quality between the two exposures of pathological findings while a statistically significant difference $(p<0.05)$ in image quality was found between the two exposures of anatomical structures.

For Groups 3 and 5, there was no statistically significant difference $(p>0.05)$ in image quality between the two exposures of either anatomical structures or pathological findings for all observers.

Table 1. Screen-digital system combinations and $\mathrm{mA}$ settings in the study.

\begin{tabular}{|l|l|c|c|c|}
\hline \multicolumn{1}{|c|}{ Combinations } & \multicolumn{1}{|c|}{$\begin{array}{c}\text { Groups } \\
(\mathrm{n}=15)\end{array}$} & $\begin{array}{c}\text { Dose } \\
\text { Reduction } \\
(\%)\end{array}$ & $\begin{array}{c}\text { First } \\
\text { Exposure } \\
(\mathrm{kV} / \mathrm{m} \mathbf{A})\end{array}$ & $\begin{array}{c}\text { Second } \\
\text { Exposure } \\
(\mathrm{kV} / \mathrm{mA})\end{array}$ \\
\hline \multirow{2}{*}{ Regular-Regular Screen } & Group 1 & 33.3 & $66 / 12$ & $66 / 8$ \\
\cline { 2 - 5 } & Group 2 & 46.6 & $66 / 12$ & $66 / 6.4$ \\
\hline \multirow{2}{*}{ Medium-Medium Screen } & Group 3 & 25 & $66 / 16$ & $66 / 12$ \\
\cline { 2 - 5 } & Group 4 & 50 & $66 / 16$ & $66 / 8$ \\
\hline Digital-Digital Radiography & Group 5 & 25 & $70 / 4$ & $70 / 3$ \\
\hline
\end{tabular}


Table 2. Evaluated anatomical structures and pathological findings.

\begin{tabular}{|c|c|}
\hline Anatomical Structures & Pathological Findings \\
\hline Anterior nasal spine & Calculus \\
\hline Articular eminence & Caries \\
\hline Condylar process & Cyst and tumour like lesions \\
\hline Coronoid process & Fracture of condyle \\
\hline Disc space & Impacted teeth \\
\hline External auditory meatus & Overextended root canal filling \\
\hline External oblique ridge & Periapical lesion \\
\hline Floor of maxillary sinus & Root fracture \\
\hline Inferior concha & Root fragment \\
\hline Inferior cortex & Strange material \\
\hline Interdental septum & Underextended root canal filling \\
\hline \multicolumn{2}{|l|}{ Mandibular canal } \\
\hline \multicolumn{2}{|l|}{ Maxillary sinus } \\
\hline \multicolumn{2}{|l|}{ Maxillary tuberosity } \\
\hline \multicolumn{2}{|l|}{ Mental foramen } \\
\hline \multicolumn{2}{|l|}{ Nasal septum } \\
\hline \multicolumn{2}{|l|}{ Periapical lamina dura } \\
\hline \multicolumn{2}{|l|}{ Periodontal ligament space } \\
\hline \multicolumn{2}{|l|}{ Styloid process } \\
\hline \multicolumn{2}{|l|}{ Zygomatic arch } \\
\hline Zygomatic bone & \\
\hline
\end{tabular}


Table 3. Results of the Mann-Whitney U test.

\begin{tabular}{|c|c|c|c|c|c|c|c|}
\hline \multicolumn{8}{|c|}{$1^{\text {st }}-2^{\text {nd }}$ Exposures } \\
\hline \multirow[b]{2}{*}{ Groups } & \multirow[b]{2}{*}{ Statistics } & \multicolumn{2}{|c|}{$1^{\text {st }}$ Observer } & \multicolumn{2}{|c|}{$2^{\text {nd }}$ Observer } & \multicolumn{2}{|c|}{$3^{\text {rd }}$ Observer } \\
\hline & & $A$ & $P$ & $A$ & $P$ & $A$ & $P$ \\
\hline \multirow{2}{*}{ Group 1} & $M-W U^{* *}$ & 81.500 & 45.000 & 49.500 & 27.000 & 82.500 & 105.000 \\
\hline & $P$ & 0.065 & $0.000^{*}$ & $0.003^{*}$ & $0.000^{*}$ & 0.073 & 0.317 \\
\hline \multirow{2}{*}{ Group 2} & $M-W U$ & 30.000 & 37.500 & 30.000 & 70.500 & 75.000 & 90.000 \\
\hline & $P$ & $0.000^{*}$ & $0.000^{*}$ & $0.000^{*}$ & 0.047 & $0.016^{*}$ & 0.073 \\
\hline \multirow{2}{*}{ Group 3} & $M-W U$ & 104.500 & 97.000 & 97.500 & 90.000 & 97.500 & 105.000 \\
\hline & P & 0.524 & 0.276 & 0.291 & 0.073 & 0.291 & 0.317 \\
\hline \multirow{2}{*}{ Group 4} & M-W U & 22.500 & 7.500 & 22.500 & 7.500 & 27.500 & 45.000 \\
\hline & P & $0.000^{*}$ & $0.000^{*}$ & $0.000^{*}$ & $0.000^{*}$ & $0.000^{*}$ & $0.001^{*}$ \\
\hline \multirow{2}{*}{ Group 5} & $M-W U$ & 104.500 & 112.500 & 112.000 & 97.000 & 112.500 & 112.500 \\
\hline & P & 0.524 & 1.000 & 0.962 & 0.276 & 1.000 & 1.000 \\
\hline
\end{tabular}

** Mann-Whitney U statistics.

* Correlation is significant at the 0.05 level

A: Anatomical structures.

$P$ : Pathological findings

For Group 4, there was a statistically significant difference $(p<0.05)$ in image quality between the two exposures of both anatomical structures and pathological findings for all observers. The results of Mann-Whitney $U$ test are shown in Table 3.

For all groups and observers, no statistically significant difference $(p>0.05)$ was found between the image quality of anatomical structures and pathological findings (Table 4).

For all groups, no statistically significant difference $(p>0.05)$ in image quality was found between the two exposures of anatomical structures and between the first exposures for pathologies in a comparison of observers. For Groups 1, 2, and 4, there was a statistically significant difference $(p<0.05)$ in image quality between the second exposures of pathologies; no statistically significant difference $(p>0.05)$ was found between the second exposures of pathologies in other groups in a comparison of observers (Table 5).

Pairwise comparisons (Mann-Whitney $U$ test) were performed between Groups 1, 2, and 4. For Groups 1 and 2, a statistically significant difference $(p<0.05)$ in image quality was found between the first and third and second and third observers. For Group 4, a statistically significant difference $(p<0.05)$ was found between the first and third observers.

A linear relationship between dose reduction and the individual ratings could not be shown so a calculated mean, standard deviation, and the mean difference between the scores of the standard and reduced $\mathrm{mA}$ images for all observers in the groups was used in the data analysis (Table 6). 
Figure 1 illustrates mean differences and the rates of tube current reduction for all observers.

\section{Discussion}

The effect of a reduction in tube current was evaluated based on image quality using medium and regular intensifying screens as well as a digital system for panoramic radiography in this study.

In the study by Dannewitz et al. ${ }^{8}$ the image quality of anatomical structures and pathological findings were assessed on digital panoramic images and the image quality of anatomical structures was assessed on various film-screen combinations and digital panoramic systems by Kaeppler et al. ${ }^{1}$ In the present study the image quality of both anatomical structures and pathological findings were assessed using two different intensifying screens for conventional panoramic radiography and a digital panoramic system based on CCD receptor.
Dula et al. ${ }^{7}$ emphasized dose reduction should be achieved by a reduction of the $\mathrm{mA}$ setting rather than the $\mathrm{kV}$ setting due to an increase in the absorption of radiation by the tissues with lower kV settings. This result was confirmed by Gijbels et al. ${ }^{9}$ For this reason, the $\mathrm{mA}$ levels were reduced while keeping the $\mathrm{kV}$ unchanged in this

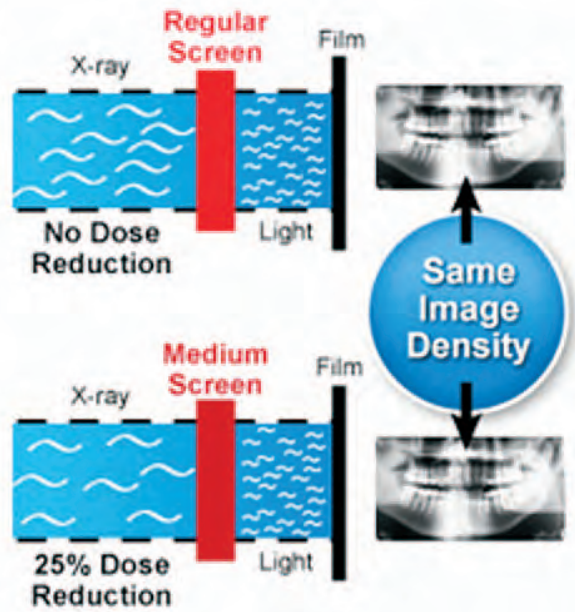

Table 4. Results of the Mann-Whitney $U$ test for anatomical structures and pathologies.

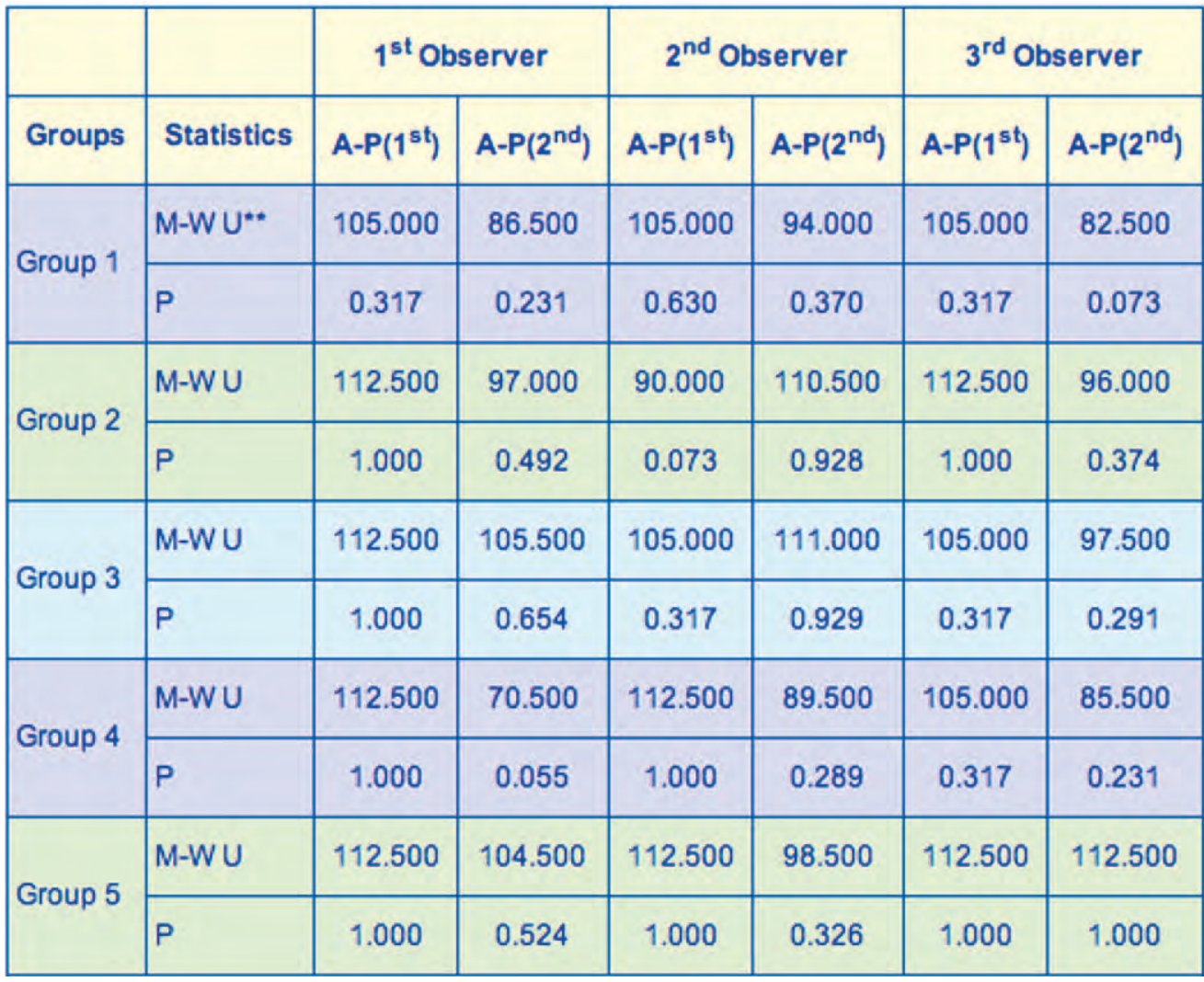

** Mann-Whitney U statistics

A: Anatomical structures

$P$ : Pathological findings 
Table 5. Results of the Kruskal-Wallis test.

\begin{tabular}{|c|c|c|c|c|c|}
\hline Groups & Statistics & $A\left(1^{\text {st }}\right)$ & $P\left(1^{\text {st }}\right)$ & $A\left(2^{\text {nd }}\right)$ & $P\left(2^{\text {nd }}\right)$ \\
\hline \multirow{3}{*}{ Group 1} & Chi-Square & 1.760 & 4.093 & 5.890 & 18.455 \\
\hline & Df & 2 & 2 & 2 & 2 \\
\hline & P & 0.415 & 0.129 & 0.053 & $0.000^{*}$ \\
\hline \multirow{3}{*}{ Group 2} & Chi-Square & .000 & 6.279 & 5.890 & 18.455 \\
\hline & Df & 2 & 2 & 2 & 2 \\
\hline & P & 1.000 & 0.050 & 0.053 & $0.000^{*}$ \\
\hline \multirow{3}{*}{ Group 3} & Chi-Square & .000 & 2.000 & .189 & 1.405 \\
\hline & Df & 2 & 2 & 2 & 2 \\
\hline & $P$ & 1.000 & 0.368 & 0.910 & 0.495 \\
\hline \multirow{3}{*}{ Group 4} & Chi-Square & 2.000 & .000 & .305 & 6.573 \\
\hline & Df & 2 & 2 & 2 & 2 \\
\hline & $P$ & 0.368 & 1.000 & 0.859 & $0.037^{*}$ \\
\hline \multirow{3}{*}{ Group 5} & Chi-Square & .000 & .000 & .539 & 1.875 \\
\hline & Df & 2 & 2 & 2 & 2 \\
\hline & $P$ & 1.000 & 1.000 & 0.764 & 0.392 \\
\hline
\end{tabular}

* Correlation is significant at the 0.05 level

Table 6. Mean standard deviation and mean difference between the scores of standard and reduced $\mathrm{mA}$ images.

\begin{tabular}{|l|c|c|c|}
\hline mA Reduction & $\begin{array}{c}\text { Standard } \\
\text { Image Mean } \\
\text { (SD) }\end{array}$ & $\begin{array}{c}\text { Reduced mA } \\
\text { Image Mean } \\
\text { (SD) }\end{array}$ & $\begin{array}{c}\text { Mean Difference } \\
\text { (SD) }\end{array}$ \\
\hline $33.3 \%$ (Group 1) & $1.08(0.269)$ & $1.59(0.669)$ & $0.511(0.076)$ \\
\hline $46.6 \%$ (Group 2) & $1.06(0.313)$ & $1.71(0.738)$ & $0.656(0.085)$ \\
\hline $25 \%$ (Group 3) & $1.05(0.214)$ & $1.21(0.493)$ & $0.167(0.059)$ \\
\hline $50 \%$ (Group 4) & $1.01(0.109)$ & $2.17(0.709)$ & $1.155(0.078)$ \\
\hline $25 \%$ (Group 5) & $1.07(0.251)$ & $1.13(0.429)$ & $0.067(0.052)$ \\
\hline
\end{tabular}




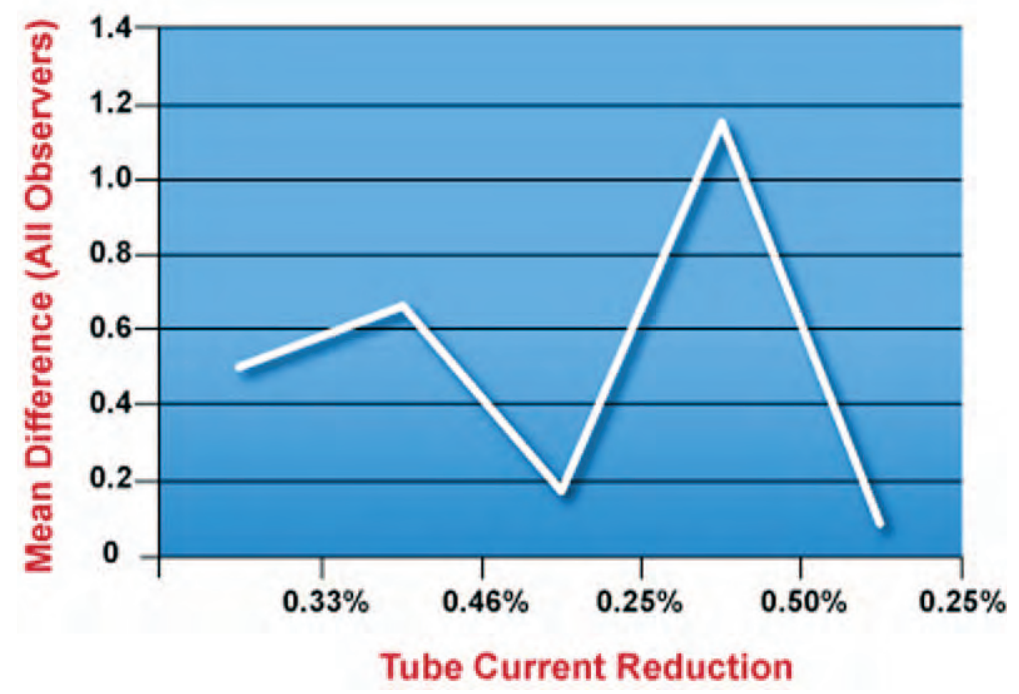

Figure 1. Mean differences and the rates of tube current reduction for all observers.

study for all groups. Because the reduction of tube current results in a decreased signal-to-noise ratio, image quality, especially high contrast, may be affected. ${ }^{10}$ However, the software associated with Trophy OP100 and Orthoralix used in this study may have compensated for this condition. Statistically significant differences $(p<0.05)$ were found between the two exposures for all observers in the groups using regular intensifying screens. It can be said dose reduction may not be achieved by only a reduction of the $\mathrm{mA}$ levels when regular intensifying screens are used. Use of low $\mathrm{kV}$ and high $\mathrm{mA}$ levels in conjunction with regular intensifying screens for dose reduction has been reported. ${ }^{1}$

In the present study there was no statistically significant difference $(p>0.05)$ between the two exposures for Group 3 (the rate of dose reduction $25 \%$ ) while a statistically significant difference $(p<0.05)$ was found in Group 4 (the rate of dose reduction $50 \%$ ) using medium intensifying screens for all observers. A dose reduction of $25 \%$ may have been achieved using medium intensifying screens. This condition is in accordance with the results of Kaeppler et al. ${ }^{1}$

There are several advantages of digital radiography such as the rapid transmission of images from one location to another, the small amount of physical storage space needed, image enhancement capabilities, and a lower environmental impact. ${ }^{411}$ In digital radiology the diagnosis frequently depends on the correct adjustment of brightness and contrast because by altering these parameters lesions can be produced or faded away. ${ }^{7}$ The observers in this study were not allowed to alter the brightness and contrast of the images using the software. The assessments were carried out under standardized conditions of the software in order to eliminate possible diagnostic differences in this study.

One of the main advantages of digital radiography is the possibility of minimizing the radiation dosage $^{12}$ with several authors $s^{6,713}$ reporting a dose reduction of about $40-70 \%$ using digital panoramic radiography compared to conventional panoramic systems. Previous studies ${ }^{5,8}$ have demonstrated the achievement of a degree of dose reduction in digital panoramic radiography without any loss of image quality. Dannewitz et al. ${ }^{8}$ reported a dose reduction up to $50 \%$ can be achieved while maintaining a satisfactory image quality and diagnostic performance. In the present study $\mathrm{mA}$ levels were reduced by $25 \%$ with no statistically significant difference between the two exposures for all observers on digital panoramic images. It can be said a $25 \%$ dose reduction was achieved for digital panoramic images.

\section{Conclusion}

According to the results of this study, dose reduction caused loss of image quality using regular intensifying screens when the tube current was reduced by $33.3 \%$ and $46.6 \%$. In further 
studies, the reduction of tube current could be limited to only $16.6 \%$.

A $25 \%$ dose reduction was achieved with a medium intensifying screen and digital panoramic radiography without any loss of image quality of either anatomical structures or pathological findings. In further studies the reduction of tube current may be reduced by $37.5 \%$ using medium intensifying screens and by $50 \%$ for digital panoramic images.

As imaging technologies continue to improve, radiologists expect future images to improve in quality while using lower radiation doses.
However, until the newest technologies are widely used, it is important to conduct studies on the effects of decreasing radiation exposures in the imaging systems currently used by the majority of clinicians.

\section{Clinical Significance}

A substantial reduction in radiation exposure can be achieved in conventional panoramic radiography using a medium intensifying screen and in digital panoramic radiography without any loss of image quality needed for radiological evaluation of anatomical structures and pathological conditions.

\section{References}

1. Kaeppler G, Dietz K, Reinert S. The effect of dose reduction on the detection of anatomical structures on panoramic radiographs. Dentomaxillofac Radiol 2006; 35(4):271-277.

2. Freeman JP, Brand JW. Radiation doses of commonly used dental radiographic surveys. Oral Surg Oral Med Oral Pathol 1994; 77(3):285-289.

3. Farman AG, Farman TT. Extraoral and panoramic systems. Dent Clin North Am 2000; 44(2):257-272.

4. Molander B, Gröndahl HG, Ekestubbe A. Quality of film-based and digital panoramic radiography. Dentomaxillofac Radiol 2004; 33(1):32-36.

5. Nelvig P, Wing K, Welander U. Sens-A-Ray. A new system for direct digital intraoral radiography. Oral Surg Oral Med Oral Pathol 1992; 74(6):818-23.

6. Haßfeld S, Ziegler C, Mühling J. Kann die digitale Panoramaschichtröntgentechnik das filmbasierte Verfahren ersetzten? Zahnärztl Welt 1997; 106:510-514.

7. Dula K, Sanderink G, van der Stelt PF, Mini R, Buser D. Effects of dose reduction on the detectability of standardized radiolucent lesions in digital panoramic radiography. Oral Surg Oral Med Oral Pathol Oral Radiol Endod 1998; 86(2):227-233.

8. Dannewitz B, Hassfeld S, Eickholz P, Mühling J. Effect of dose reduction in digital dental panoramic radiography on image quality. Dentomaxillofac Radiol 2002; 31(1):50-55.

9. Gijbels F, Sanderink G, Bou Serhal C, Pauwels H, Jacobs R. Organ doses and subjective image quality of indirect digital panoramic radiography. Dentomaxillofac Radiol 2001; 30(6):308-313.

10. Chesters MS. Human visual perception and ROC methodology in medical imaging. Phys Med Biol 1992; 37(1):1433-1476.

11. Mastoris M, Li G, Welander U, McDavid WD. Determination of the resolution of a digital system for panoramic radiography based on CCD technology. Oral Surg Oral Med Oral Pathol Oral Radiol Endod 2004; 97(3):408-414.

12. Hildebolt CF, Fletcher G, Yokoyama-Crothers N, Conover GL, Vannier MW. A comparison of the response of storage phosphor and film radiography to small variations in X-ray exposure. Dentomaxillofac Radiol 1997; 26(3):147-151.

13. Gijbels F, Jacobs R, Bogaerts R, Debaveye D, Verlinden S, Sanderink G. Dosimetry of digital panoramic imaging. Part I: patient exposure. Dentomaxillofac Radiol 2005; 34(3):145-149. 
About the Authors

\section{Moryom Toraman Alkurt, DDS, PhD}

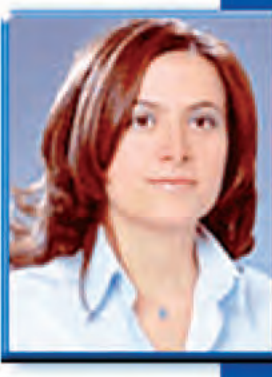

Dr. Alkurt is an Assistant Professor in the Department of Oral Diagnosis and Radiology of the Faculty of Dentistry at Gazl University in Ankara, Turkey. Her research interest is in dentomaxillofacial imaging systems and oral medicine.

e-mail: mtalkurt@gmail.com

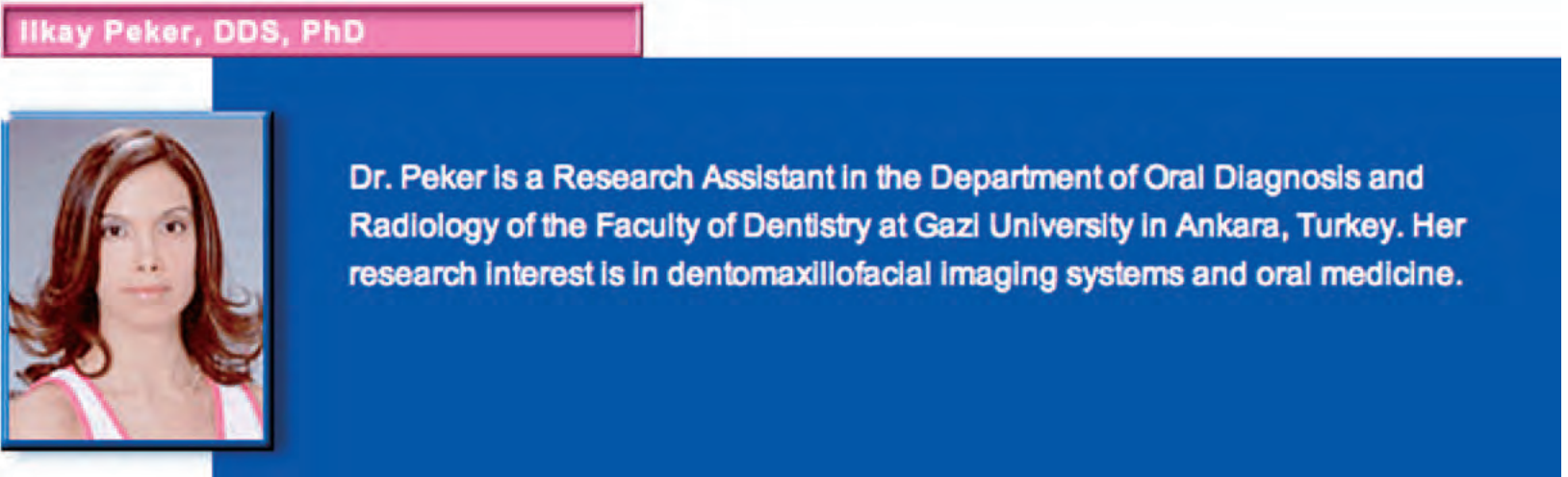

Guiten Usalan, DDS, PhD

Dr. Usalan is an oral radiologist at the Health Clinics of the Municipality of Çankaya in Ankara, Turkey.

Bulent Altunkaynak, BDS, PhD

Dr. Altunkaynak is an Assistant Professor in the Department of Statistics, Faculty of Arts and Sciences at the Gazl University in Ankara, Turkey. His research interest is In biostatistics.

\section{Acknowledgement}

This study was presented at the 12th BaSS Congress on April 12-14, 2007 in Istanbul, Turkey. 